\title{
AN ANALYTICAL SOLUTION OF THE PROBLEM OF PLASMA EJECTION FROM THE MAGNETOSPHERE OF AN AXISYMMETRIC ROTATOR
}

\author{
S. V. Bogovalov \\ Moscow Engineering Physics Institute
}

\begin{abstract}
The flow of $e^{+} e^{-}$plasma ejected by an axisymmetrically rotating magnetized neutron star is considered in a hydrodynamical approximation. It is shown that in the vicinity of the light cylinder a helical discontinuity is formed. The transformation of toroidal magnetic field energy into plasma energy takes place at this discontinuity. Particles are accelerated to an energy of $10 \mathrm{TeV}$ for a neutron star with the characteristics of the Crab pulsar.
\end{abstract}

\section{Introduction}

The problem of plasma ejection from the magnetosphere of an axisymmetric rotator is of great importance for understanding the charged particle acceleration and electromagnetic radiation processes in pulsars. The magnetospheric properties of an axisymmetric rotator were discussed by Goldreich and Julian (1969). Within the framework of this model, Beskin, Gurevich and Istomin (1983) have explored the possibility of strong particle acceleration close to the light cylinder.

The purpose of this paper is to investigate the movement of plasma as it emerges from the surface of the neutron star and streams out to large distances. In particular, the characteristics of plasma motions near the velocity-of-light cylinder are of great interest.

We suppose that near the surface of the neutron star a sufficiently dense $e^{+} e^{-}$plasma is produced and that beyond this point there is no further production of particles in the magnetosphere (Ruderman and Sutherland 1975, Gurevich and Istomin 1985b).

\section{Basic equations}

The stationarity of the plasma flow and the azimuthal symmetry of the problem enable us to write down easily the two first integrals of motion in the hydrodynamical approximation. They are derived from the conservation of the energy-momentum tensor $d T^{i k} / d x^{k}=0$. The energy-momentum tensor $T^{i k}$ is the sum of the tensors pertaining to material particles and fields. According to Landau and Lifshitz (1973), the term describing material particles is

$$
T_{a}^{i k}=\left(\begin{array}{ll}
m \gamma c^{2} n\left[\varepsilon+p(v / c)^{2}\right] & S / c \\
S / c & \sigma^{\alpha \beta}
\end{array}\right),
$$

where

$$
S / c=(\varepsilon+p) n \gamma \boldsymbol{v} m c
$$

and

$$
\sigma^{\alpha \beta}=\gamma(\varepsilon+p) n m v^{\alpha} v^{\beta}+p n m c^{2} \delta^{\alpha \beta} / \gamma .
$$

The term describing fields is

$$
T_{f}^{i k}=\left(\begin{array}{ll}
\left(E^{2}+H^{2}\right) / 8 \pi & S / c \\
S / c & \sigma^{\alpha \beta}
\end{array}\right)
$$

where

$$
\begin{gathered}
S / c=[\boldsymbol{E} \times \boldsymbol{H}] / 4 \pi \\
\sigma^{\alpha \beta}=\left(-E^{\alpha} E^{\beta}-H^{\alpha} H^{\beta}+\frac{1}{8 \pi} \delta^{\alpha \beta}\left(E^{2}+H^{2}\right)\right),
\end{gathered}
$$

$\varepsilon$ and $p$ are the energy and pressure per particle (in $m c^{2}$ units) in coordinates related to the center of mass, and $n$ is the density of particles in the plasma. These equations are combined on the condition that the fields are effectively "frozen" into the plasmathat is, in the limit of infinitely high conductivity (Landau and Lifshitz 1982)

$$
\boldsymbol{E}+[\boldsymbol{v} \times \boldsymbol{H}] / c=0 .
$$

Then we can write down Maxwell's equations and the continuity relation

$$
\begin{aligned}
& \boldsymbol{\nabla} \times \boldsymbol{E}=\mathbf{0} \\
& \boldsymbol{\nabla} \cdot \boldsymbol{H}=\mathbf{0} \\
& \boldsymbol{\nabla} \cdot \boldsymbol{n} \boldsymbol{v}=\mathbf{0}
\end{aligned}
$$

To define the plasma flow accurately it is necessary to know all the electric and magnetic fields within the magnetosphere. However, it turns out that one can obtain a general solution to the problem for an arbitrary poloidal field which has the same topology as the Goldreich and Julian (1969) model. For this purpose it is convenient to consider the plasma flow in a special curvilinear system of coordinates given by the poloidal field. 
Description of the coordinate system

Let us introduce an orthogonal coordinate system so that one of its axe's $\boldsymbol{e}_{\alpha}$ coincides with the direction of the poloidal magnetic field line $\boldsymbol{H}_{\alpha}$, the second axis $\boldsymbol{e}_{\varphi}$ corresponds to the azimuthal angle $\varphi$, and the third axis is $e_{\xi}=e_{\alpha} \times e_{\varphi}$. The curvilinear coordinates are then $\alpha, \varphi, \xi$, and the magnetic field in this system of coordinates has the form

$$
\boldsymbol{H}=\boldsymbol{e}_{\alpha} H^{\alpha}+\boldsymbol{e}_{\varphi} H^{\varphi} .
$$

The real vector in such a coordinate system is $H^{\alpha} / \sqrt{g}_{\alpha}, H^{\varphi} / \sqrt{g}_{\varphi}$, where $g_{\alpha}, g_{\varphi}$ are the components of the metric tensor, $g_{\varphi}$ being $\rho^{2}$, and $\rho$ the distance to the stellar rotation axis. The velocity will be

$$
v=e_{\alpha} v^{\alpha}+e_{\varphi} v^{\varphi}+e_{\xi} v^{\xi}
$$

From the condition $\nabla \times E=0$, it follows that $E^{\varphi}=0$. Recalling the "frozen" condition on the field gives $v^{\xi}=0$. From eqs.(3), (5) and (6) it follows that the electric field has only one non-zero component $E=E^{\xi}$.

Determining the form of the metric coefficient $g_{\xi}$, the equation $\boldsymbol{\nabla} \cdot \boldsymbol{H}=0$ in the curvilinear system of coordinates will be

$$
(1 / \sqrt{g}) \frac{\partial}{\partial \alpha}\left(\sqrt{g} H^{\alpha} / \sqrt{g_{\alpha}}\right)=0 .
$$

From the above we obtain

$$
\sqrt{g_{0 \xi}(\xi) / g_{\xi}(\alpha, \xi)}=\rho H^{\alpha} / \rho_{0} H_{0}^{\alpha},
$$

where $g$ is the determinant of the metric tensor, and the index " 0 " is evaluated at the stellar surface.

\section{Solution}

As a result of the energy and angular-momentum flux conservation laws, we have the two first integrals of motion in a curvilinear system of coordinates

$$
\begin{aligned}
J(\xi) m c \gamma(\varepsilon+p)-\sqrt{g_{\varphi} g_{\xi}} E H^{\varphi} / 4 \pi & =\tilde{W}(\xi) \\
\sqrt{g}_{\varphi} m v^{\varphi} \gamma J(\xi)(\xi+p)-\sqrt{g_{\varphi} g_{\xi}} H^{\alpha} H^{\varphi} / 4 \pi & =\tilde{M}(\xi),
\end{aligned}
$$

where $J(\xi)=\sqrt{g_{\varphi} g_{\xi}} n v^{\alpha}$ is the plasma flux, $\tilde{W}(\xi)$ the energy flux, and $\tilde{M}(\xi)$ the angular momentum flux. All three are constant on a field line of constant $\xi$.

Introducing new variables

$$
\begin{aligned}
\tilde{\mathbf{X}} & =\rho \Omega / c, \\
\Gamma & =\gamma(\varepsilon+p), \\
U_{\alpha} & =\Gamma v^{\alpha} / c, \\
U_{\varphi} & =\Gamma v^{\varphi} / c, \text { and } \\
h & =H^{\varphi} / H^{\alpha}
\end{aligned}
$$

the dependence $E=\tilde{X} q(\xi) H$ can be derived from eqs.(4) and (7), where $q(\xi)$ is an arbitrary function, constant on the poloidal field line (for more details, see Bogovalov 1989).

On changing to the new variable $X=\tilde{X} q(\xi)$, we obtain a set of algebraic equations from eqs.(1-10)

$$
\begin{aligned}
\Gamma-G h & =W(\xi) \\
x U_{\varphi}-G h & =M(\xi) \\
x \Gamma+U_{\alpha} h & =U_{\varphi} \\
\Gamma^{2} & =(\varepsilon+p)^{2}+U_{\alpha}^{2}+U_{\varphi}^{2},
\end{aligned}
$$

where the function $G(\alpha, \xi)$ is equal to

$$
G(\alpha, \xi)=\frac{\left(H_{0}^{\alpha}\right)^{2} X_{0}}{4 \pi n_{0} m c v_{0}^{\alpha}} \frac{X H^{\alpha}}{X_{0} H_{0}^{\alpha}} .
$$

A single equation defining $h$ as a function of the parameter $x$ can be obtained from the set of relations in eq.(11)

$$
\left(x \sqrt{\frac{v^{2}-(\varepsilon+p)^{2}(1-X)^{2}}{\left(1+h^{2}-X^{2}\right)}}-G\left(1-X^{2}\right)\right) h=M-X^{2} W
$$

where $V=W-M$. All other quantities are expressed by the relations

$$
\begin{aligned}
U_{\alpha} & =\sqrt{\frac{v^{2}-(\varepsilon+p)^{2}(1-X)^{2}}{\left(1+h^{2}-X^{2}\right)}} \\
\Gamma & =\frac{\left(V+X h U_{\alpha}\right)}{\left(1-X^{2}\right)} \\
U_{\varphi} & =\frac{(\Gamma-V)}{X} .
\end{aligned}
$$

For the sake of definiteness, we will assume that the rotation axis and magnetic moment are parallel. We are interested in solutions for which $\Gamma>1, U_{\alpha}>$ 0 , and $h<0$. The last two inequalities pertain to the matter flux and electromagnetic energy flux directed from the star.

Figure 1 schematically illustrates the respective behavior of the right-hand and left-hand sides of eq.(13) for the cases when $X<X_{*}$ (solid lines), $1>X>X_{*}$ (dashed lines), and $X>1$ (dotted lines), where $X_{*}=\sqrt{M / W}$. It is obvious that at $X<X_{*}$ there is only one solution of eq.(13). We refer to it as $A$. It describes the plasma flow from the stellar surface. At $X>X *$ another root $B$ appears, which also describes a physically admissible solution. The analysis shows (Bogovalov 1990) that for the $A$ solution the inequality

$$
\left(v^{\alpha} / c\right)^{2}<\left(H^{2}-E^{2}\right) / 4 \pi m c^{2} n \gamma(\varepsilon+p)
$$

is realized. For the $B$ solution, the inequality of eq.(15) is just reversed. The azimuthal magnetic field in the flow $A$ is thus energetically favored. This 


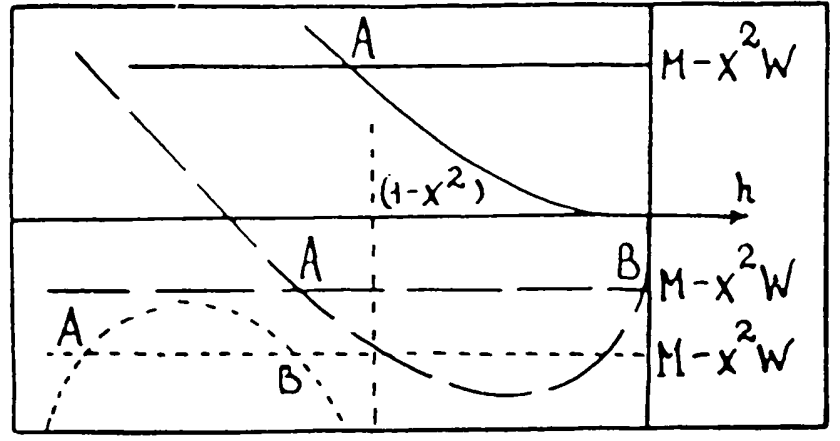

Figure 1 See text

is because the electric current emanating from the polar cap of the pulsar flows along the magnetic field lines. Magnetic systems in which electric current flows in the magnetic field direction are known to be unstable with respect to helical disturbances (Kadomtzev 1988). Therefore, it is reasonable to assume that at large distances from the pulsar the physically realized plasma flow will be described by root $B$. The transition from flow $A$ to flow $B$ must then be discontinuous.

The analysis carried out by Bogovalov (1990b) has shown that a stable discontinuity can occur only at the point $X_{*}$ and that it will be helical in form
(Landau and Lifshitz 1982). At this discontinuity the magnetic field vector rotates around the normal to break the surface, and the plasma temperature $\varepsilon$ does not change. In this case the most important feature is the transformation at the discontinuity of azimuthal magnetic field energy into relativistic particle energy. After the break the azimuthal magnetic field returns to zero. For stellar parameters appropriate for the Crab pulsar PSR 0531+21, it appears that particles will be accelerated up to $5 \times 10^{7} m c^{2}$ at the discontinuity. The total energy released in these particles will be

$$
L=H^{2} R^{6} \Omega^{4} / 4 c^{3},
$$

(Beskin, Gurevich, and Istomin 1983b), and for PSR $0531+21$ this value will of the order $3 \times$ $10^{38} \mathrm{erg} \mathrm{s}^{-1}$. This is quite sufficient to account for the energy released into the Crab Nebula in the form of relativistic particles (Shklovskii 1970).

The particle acceleration at the discontinuity is produced as usual by an electric field. At the discontinuity the particles move transverse to the magnetic field, accumulate energy and simultaneously form a current which shorts the current flowing out of the polar cap region of the pulsar. 